\title{
Promoción de la investigación: viviendo sin una cultura de publicación
}

\author{
Rodrigo M. Carrillo-Larco, Jorge Osada
}

En los últimos años se ha observado un aumento de la producción científica nacional en pregrado. Si bien la producción científica en pregrado es heterogénea y desarrollada principalmente por algunas instituciones educativas [1], resulta notable mencionar que en el período 2009-2010, en el 12,9\% de las publicaciones originales en revistas indizadas en SciELO-Perú ha participado un estudiante de medicina como autor [2].

El objetivo de la presente carta es reflexionar sobre los motivos que limitan dichas publicaciones. Se han hecho avances importantes en la promoción de la investigación, a través de becas y fondos concursables, que han afectado positivamente el interés de estudiantes y docentes para producir información pertinente a nuestra realidad. A pesar de ello, ¿por qué no se ven más publicaciones?

A lo largo de la carrera profesional de medicina humana se desarrollan diversos cursos y talleres, como parte del currículo regular o como electivos, en los cuales los participantes adquieren habilidades básicas para la elaboración y ejecución de un proyecto de investigación. Estos cursos son importantes porque invitan a los estudiantes al campo científico, pero en la mayoría de casos no se convierten de un ejercicio práctico a una contribución tangible en la realidad $[3,4]$.

No todos los alumnos consideran que el epílogo de un trabajo de investigación es la publicación y que, por tanto, sus trabajos pueden o deben publicarse $[5,6]$. Resulta común encontrarse con la creencia de que la calidad y el rigor de la revisión de los estudios producidos por alumnos es o debe ser inferior al de los profesionales [6]. En general, dejarse llevar por esta idea representa bajar el nivel de las producciones científicas estudiantiles y genera profesionales con una base deficiente que no pueden cumplir con los estándares científicos requeridos y ven la investigación como una meta inalcanzable.

El problema es la ausencia de 'cultura de publicación' [3,4]. Hemos aceptado que la investigación es útil y debemos practicarla activamente; sin embargo, continuamos viendo la investigación como un ejercicio teórico que debe conocerse, aprenderse y almacenarse. Se olvida el impacto de la difusión de información, dándole mayor importancia a su desarrollo y presentando datos en comunicaciones institucionales internas, de baja trascendencia. Esto obliga a otros investigadores o instituciones a realizar esfuerzos similares, lo que genera una perdida evitable de recursos.

Para afrontar este problema resulta importante emprender acciones claras y concretas con planes a largo y medio plazo a diferentes niveles. Se debe modificar el pensamiento, actitudes y normas, tanto individuales como institucionales, para facilitar y favorecer la producción científica de calidad, estimulando a los jóvenes investigadores a dar los primeros pasos de un futuro prolífico.

Los alumnos que realizan algún trabajo de investigación deben saber que pueden publicarlo y ser asesorados con este fin. No todos los trabajos podrán transformarse en un artículo original, pero todos tienen el potencial, si son guiados de forma adecuada, de convertirse en una publicación viable que genere nuevo conocimiento para nuestra sociedad.

Bibliografía

1. Huamaní C, Chávez-Solís P, Mayta-Tristán P. Aporte estudiantil en la publicación de artículos científicos en revistas médicas indizadas en SciELO-Perú, 1997-2005. An Fac Med 2008; 69: 42-5.

2. Taype-Roldán A, Lajo-Aurazo Y, Gutiérrez-Brown R, ZamalloaMasías N, Saldaña-Gonzales M. Aporte de las sociedades estudiantiles en la publicación científica en SciELO-Perú, 2009-2010. Rev Peru Med Exp Salud Publica 2011; 28: 688-99.

3. Alarcón-Villaverde J, Romaní F, Gutiérrez C. Publicaciones científicas estudiantiles producidas en el curso de Epidemiología de la Facultad de Medicina de la Universidad Nacional Mayor de San Marco durante el periodo 2003-2009. An Fac Med 2010; 71: 111-6.

4. Arriola-Quiroz I, Curioso WH, Cruz-Encarnación M, Gayoso O. Characteristics and publication patterns of these from a Peruvian medical school. Health Info Libr J 2010; 27 : 148-54.

5. Mayta-Tristán P, Peña-Oscuvilca A. Importancia de la publicación en las sociedades científicas de estudiantes de medicina del Perú: estudio preliminar. CIMEL 2009; 14: 27-34.

6. Gutiérrez C, Mayta P. Publicación desde el pregrado en Latinoamérica: importancia, limitaciones y alternativas de solución. CIMEL 2003; 8: 54-60.
Sociedad Científica de Estudiantes de Medicina Cayetano Heredia (R.M. Carrillo-Larco). Unidad de Epidemiología Clínica; Facultad de Medicina Alberto Hurtado; Universidad Peruana Cayetano Heredia (J. Osada); Lima, Perú.

Correspondencia:

Rodrigo M. Carrillo Larco. Avda. 28 de Julio, 818. Dpto. 601 Lima 18, Perú.

E-mail:

rodrigo.carrillo@upch.pe

C) 2012 Educación Médica 